\title{
TAME NONSMOOTH INVERSE MAPPING THEOREMS
}

\author{
TOSHIZUMI FUKUI, KRZYSZTOF KURDYKA, AND LAURENTIU PAUNESCU
}

\begin{abstract}
We give several versions of local and global inverse mapping theorem for tame non necessarily smooth, mappings. Here tame mapping means a mapping which is subanalytic or, more generally, definable in some o-minimal structure. Our sufficient conditions are formulated in terms of various properties (convexity, positivity of some principal minors, contractiblity) of the space of Jacobi's matrices at smooth points.
\end{abstract}

\section{Introduction.}

The classical inverse mapping theorem gives conditions under which a $C^{r}, r \geq$ 1 mapping admits locally a $C^{r}$ inverse. F. H. Clarke [2] generalized the inverse mapping theorem to merely Lipschitz mappings. For a Lipschitz map-germ $f$ : $\left(\mathbf{R}^{n}, 0\right) \rightarrow\left(\mathbf{R}^{n}, 0\right)$ he defined the generalized Jacobian $\partial f(0)$ as the convex hull of all matrices which are limits of Jacobi's matrices $d f(x)$ as $x \rightarrow 0$. Then he showed that $f$ admits Lipschitz inverse when $\partial f(0)$ does not contain singular matrices.

In this paper we show several versions of inverse mapping theorems for nonsmooth mappings, which belong to a tame category. Our results apply also to smooth mappings, with assumptions weaker than the classical ones we obtain existence of not necessarily smooth inverses. For a convex open subset $U$ of $\mathbf{R}^{m}$, we say that a mapping $f: U \rightarrow \mathbf{R}^{n}$ is tame, if it is subanalytic or, more generally, definable in some o-minimal structure. We recall the definition of subanalytic mappings and definable mappings in an o-minimal structure in \&4 For instance all semi-algebraic maps are tame, in particular all rational maps are tame.

In Section 3 we investigate Clarke's idea, of taking the convex hull of all Jacobi's matrices, and we state three global inverse mapping (more precisely global injectivity) Theorems 3.2, 3.3 and 3.4. We also state two local inverse Theorems 3.11 and 3.13 based on a study of some minors of Jacobi's matrices. In this section we give also several important and relevant examples which illustrate the relations between our various inverse mapping theorems. The remaining part of the paper is organized as follows.

In Section 2 we recall all necessary material from convexity theory. In Section 4 we state and show a key property of tame maps which is crucial in the proof of our theorems.

In Sections 5 and 6 we prove Theorems 3.2 , 3.3, 3.4 and respectively Theorems 3.11 and 3.13 .

Date: 17 December 2007.

2000 Mathematics Subject Classification. Primary 52Axx, 32F27, 03C64, Secondary 14P10, $58 \mathrm{~A} 35$.

Key words and phrases. inverse mapping, univalence, convex, subanalytic, o-minimal, Lipschitz. 
In Section 7, we investigate the contractibility of the set of all Jacobi's matrices in the general linear group $\mathrm{GL}(n, \mathbf{R})$, as a possible sufficient condition for the local invertibility. In the case $n=2$, precisely we observe the the following fact. Let $f:\left(\mathbf{R}^{2}, 0\right) \rightarrow\left(\mathbf{R}^{2}, 0\right)$ be a continuous tame mapping which is a local diffeomorphism, except possibly at the origin. If the mapping

$$
d f:\left(\mathbf{R}^{2}-0,0\right) \rightarrow \mathrm{GL}(2, \mathbf{R})
$$

is null homotopic, then $f$ is a homeomorphism. We give also an example which shows that this statement is wrong for $n=3$.

The problem of finding sufficient conditions for the injectivity of continuous maps has attracted numerous mathematicians working in different fields. In the paper we have essentially cited only a few relevant contributions. An excellent overview of related recent results can be found for instance in [5] and also in [6].

\section{Preliminaries}

2.1. Convexity. We recall here basic notions and facts from the convexity theory, needed in this paper. Let $V$ be a normed space (of finite dimension) and let $A \neq \emptyset$ be a subset of $V$. We denote by af $(A)$ the affine hull of $A$, that is, the smallest affine subspace of $V$ which contains $A$. Recall that the relative interior, denoted by ri $(C)$, of a set $C \subset V$ is the interior of $C$ in its affine hull af $(C)$. If $C$, a convex set, lies in the both sides of a hyperplane $\pi$, then $\operatorname{ri}(C) \cap \pi=\operatorname{ri}(C \cap \pi))$, we use this several times in our examples.

By $\operatorname{co}(A)$ we denote the convex hull of $A$, that is, the smallest convex subset of $V$ which contains $A$. By $\overline{c o}(A)$ we denote the closed convex hull of $A$, that is, the smallest closed and convex subset of $V$ which contains $A$. Recall that $\overline{c o}(A)=$ $\overline{c o}(\bar{A})=\overline{c o(A)}$ and, if $C$ is convex, $\operatorname{ri}(C)=\operatorname{ri}(\bar{C})$. In general $c o(\bar{A}) \subseteq \overline{c o}(A)$.

Let $A$ be a subset of $V$, we say that a subset $S$ of $A$ is extremal if no point of $S$ is an interior point of a segment with endpoints in $A$, except the case where both extremities belong to $S$. This can be formally stated as follows: for any $x, y \in A$ and any $t \in(0,1)$, if

$$
t x+(1-t) y \in S
$$

then $x, y \in S$. If $S=\{a\}$ is extremal, then we say that $a$ is an extremal point of $A$. We introduce also a weaker notion of extremality. We say that a subset $S$ of $C$ is semi-extremal in $C$ if and only if $C \backslash S$ is convex.

We state now several elementary facts about the above sets.

Lemma 2.1. Let $S$ be an extremal (respectively semi-extremal) subset in $C$ and $C^{\prime} \subset C$. Then $S \cap C^{\prime}$ is an extremal (respectively semi-extremal) subset in $C^{\prime}$.

Lemma 2.2. Any extremal subset is also a semi-extremal subset. In general the converse is not true, it is true for one point sets, that is, any semi-extremal point is an extremal point.

Lemma 2.3. If $C \subset V$ is convex and $S \subsetneq C$ is extremal in $C$, then $S \cap$ riC $=\emptyset$.

We need also a lemma about the image of an extremal set. 
Lemma 2.4. Let $\varphi: V \rightarrow W$ be a linear map. Assume that $C \subset V$ is convex and $S \subset C$ is extremal (respectively semi-extremal) in $C$, moreover $S=C \cap \varphi^{-1}(\varphi(S)$ ). Then $\varphi(S)$ is extremal (respectively semi-extremal) in $\varphi(C)$.

Proof. Indeed $\varphi(C) \backslash \varphi(S)=\varphi(C \backslash S)$, by our assumption. Hence by Lemma 2.2 the set $\varphi(S)$ is extremal in $\varphi(C)$.

2.2. Closed convex cones. Let $V$ be a normed space of finite dimension and let $X$ be a subset of $V$. We say that $X$ is a cone if $x \in X$ and $t \geq 0$ implies $t x \in X$. For any $A \subset V$ we denote by $\overline{c o n e}(A)$ the closed convex conic hull of $A$, that is the smallest closed convex cone in $V$ which contains $A$. We denote by $\bar{B}(1)$ the closed unit ball in $V$. We have the following obvious fact.

Lemma 2.5. Let $X \subset V$ be a cone, then $X$ is closed if and only if $X \cap \bar{B}(1)$ is compact.

Indeed the map $\mathbf{R}_{\geq 0} \times(X \cap \bar{B}(1)) \ni(t, x) \mapsto t x \in X$ is surjective and proper.

Lemma 2.6. Let $V, W$ be linear spaces of finite dimension and let $\varphi: V \rightarrow W$ be a linear map. Assume that $X \subset V$ is a closed convex cone. Then $\varphi(X)$ is also a closed convex cone.

Proof. It easy to see that $\varphi(X)$ is a convex cone, we prove now that it is also closed. Indeed we have

$$
\varphi(X)=\overline{c o n e}(\varphi(X \cap \bar{B}(1)),
$$

so the claim follows from Lemma 2.5, since $\varphi(X \cap \bar{B}(1))$ is compact.

2.3. Extremal sets in the space of matrices. We shall use frequently the extremal property with respect to some subspaces in the space of matrices. Let $X$ be a convex subset of the space of matrices $M(m, n)$. For a vector $v \in \mathbf{R}^{n},|v|=1$ we put

$$
\Sigma_{v}(X):=\{A \in M(m, n): A v=0\} \cap X .
$$

Proposition 2.7. The following conditions are equivalent:

(1) for each $v \in \mathbf{R}^{n},|v|=1$ the set $\Sigma_{v}(X)$ is extremal (or semi-extremal) in $X$

(2) for any $A, B \in X$ we have

$$
\operatorname{ker}(A+B) \subseteq \operatorname{ker} A .
$$

Proof. First we observe that $\Sigma_{v}(X)$ is semi-extremal in $X$ if and only if

$$
\varphi(X) \cap \varphi(-X) \subseteq\{0\}
$$

where $\varphi: M(m, n) \rightarrow \mathbf{R}^{n}, \varphi(A)=A v$. Now we note that $v \in \operatorname{ker}(A+B)$ if and only if $A v$ and $B v$ are opposite, and obtain the equivalence using inclusion (1). 
2.4. Distance to singular matrices. Let $M(m, n)$ denote the set of $m \times n$ matrices and $\Sigma \subset M(m, n)$ the set of singular matrices. We consider $M(m, n)$ as the space of linear maps from $\mathbf{R}^{m}$ to $\mathbf{R}^{n}$ equipped with the operator norm induced by some fixed norms on $\mathbf{R}^{m}$ and $\mathbf{R}^{n}$. We first recall two useful facts which we need later.

Lemma 2.8. Assume that $m \leq n$, then for any $A \in M(m, n)$ we have

$$
\nu(A):=\operatorname{dist}(A, \Sigma)=\inf \left\{|A v|: v \in S^{m-1}\right\} .
$$

Moreover if $m=n$, then $\nu(A)=\left\|A^{-1}\right\|^{-1}$.

Proof. See Proposition 2.2 in [12].

Remark 2.9. Usually for given matrix $A$ the computation of $\nu(A)=\operatorname{dist}(A, \Sigma)$ is not obvious. Often it is enough and more efficient to use an auxiliary function $g$ with the property that, there exist constants $a, b>0$ such that $a g(A) \leq \nu(A) \leq b g(A)$, for any matrix $A$. For instance we can take

$$
g(A)=\max _{I} \frac{\left|\operatorname{det} A_{I}\right|}{\left(\sum_{i, j} A_{I, i, j}^{2}\right)^{1 / 2}},
$$

where $A_{I}$ are the $m \times m$ matrices extracted from $A$, and $A_{I, i, j}$ denotes the determinant (minor) of the matrix $A_{I}$ obtained from $A$ by deleting the $i$-th row and the $j$-th column.

For further reference we mention here the classical Theorem of the Invariance of Domain.

Lemma 2.10 (Invariance of Domain). Let $U$ be an open subset of $\mathbf{R}^{n}$. Then, every injective continuous mapping $f: U \rightarrow \mathbf{R}^{n}$ is open.

Proof. See [3, IV. Proposition 7.4].

2.5. Notations. Let $U$ be an open subset of $\mathbf{R}^{m}$ and $f: U \rightarrow \mathbf{R}^{n}$ be a continuous tame mapping. We denote by $B(f)$ the set of points where $f$ is not differentiable.

Let $V$ be an open subset of $U$, we denote by

$$
c o(f, V):=c o(\mathcal{D}(f, V)), \overline{c o}(f, V):=\overline{c o}(\mathcal{D}(f, V))
$$

the convex hull and the closed convex hull respectively, of the set

$$
\mathcal{D}(f, V):=\{d f(x): x \in V-B(f)\} .
$$

For short we shall write $c o(f)$ and $\overline{c o}(f)$ instead of $c o(f, U)$ and of $\overline{c o}(f, U)$ respectively. Also we use $\mathcal{D}(f)$ instead of $\mathcal{D}(f, U)$, and for a given $v \in \mathbf{R}^{n}$, we put $\mathcal{D}_{v}(f):=\{A v: A \in \mathcal{D}(f)\}$. Finally we consider the closed convex cone generated by $\mathcal{D}(f)$, and write

$$
\overline{\text { cone }}(f):=\overline{\text { cone }}(\mathcal{D}(f)) .
$$

Clearly $\overline{\text { cone }}(f)=\overline{\text { cone }}(\operatorname{co}(f))=\overline{\text { cone }}(\overline{c o}(f))$.

The following routine property (Koopman-Brown theorem cf. [13]) of tame sets will be useful.

Lemma 2.11. Let $B \subset \mathbf{R}^{m}$ be a set definable in an o-minimal structure. Assume that $B$ is nowhere dense in $\mathbf{R}^{m}$. Then for each $x \in \mathbf{R}^{n}$ the set $B(x)=\left\{y \in \mathbf{R}^{m}\right.$ : $[x, y] \cap B$ is finite $\}$ is dense and definable in $\mathbf{R}^{m}$. 


\section{Results and Examples}

Let us first state the result of F.Clarke [2] which has inspired our work. Recall that if $U \subset \mathbf{R}^{n}$ is open and $f: U \rightarrow \mathbf{R}^{n}$ is a Lipschitz map, then $f$ is almost everywhere differentiable, hence the set the $U \backslash B(f)$ is dense in $U$. We define $\overline{c o}(f)$ as in Section 2 .

Theorem 3.1 (Clarke). Let $U$ be a convex open subset of $\mathbf{R}^{n}$ and let $f: U \rightarrow \mathbf{R}^{n}$ be a Lipschitz map. Assume that $\overline{c o}(f) \cap \Sigma=\emptyset$, then $f$ is injective, and moreover $f^{-1}$ is Lipschitz.

Our first global injectivity theorem is the following.

Theorem 3.2. Let $U$ be a convex open subset of $\mathbf{R}^{m}$. Assume that $f: U \rightarrow \mathbf{R}^{n}$, $m \leq n$, is tame and continuous. If $\operatorname{dist}(\operatorname{co}(f), \Sigma) \geq \delta$, then

$$
\left|f\left(x^{\prime}\right)-f(x)\right| \geq \delta\left|x^{\prime}-x\right| \quad \text { for all } x, x^{\prime} \in U .
$$

In particular $f$ is injective. If $m=n$, then $f(U)$ is open, $f: U \rightarrow f(U)$ is a homeomorphism and $f^{-1}$ satisfies the Lipschitz condition with constant $\delta^{-1}$.

Note that Theorem 3.2 implies Theorem 3.1. Indeed, if $f$ is Lipschitz the set $\overline{c o}(f)$ is compact, hence the condition $\overline{c o}(f) \cap \Sigma=\emptyset$ implies that there exists $\delta>0$ such that $\operatorname{dist}(c o(f), \Sigma) \geq \delta$. In the category of tame maps Theorem 3.2 is stronger than Clarke's theorem, since we do not assume that $f$ is Lipschitz or even locally Lipschitz. Actually both theorems can be seen as non-smooth variants (with Lipschitz inverse) of the celebrated Hadamard's theorem, see [7], for $C^{1}$ maps.

In the next theorem we will assume that that Clarke's type assumption is verified only generically.

Theorem 3.3. Let $U$ be a convex open subset of $\mathbf{R}^{n}$ and $f: U \rightarrow \mathbf{R}^{n}$ a continuous tame mapping. Assume that there exists a nowhere dense closed $B \subset U$, such that $f$ is a $C^{1}$ immersion on $U \backslash B$, that is, $d f(x)$ is non-singular for any $x \in U \backslash B$. Suppose that $f$ satisfies the following conditions:

$\left(C_{r}\right) \Sigma \cap \operatorname{co}(f, U \backslash B)=\emptyset$, where $\operatorname{co}(f, U \backslash B):=\operatorname{co}\{d f(x): x \in U \backslash B\}$;

(I) $f$ is injective on $B$.

Then $f$ is injective.

Surprisingly, in the next theorem we allow some singular matrices in $\overline{c o}(f)$. For any vector $v \in \mathbf{R}^{n},|v|=1$, we put

$$
\Sigma_{v}(f):=\{A \in M(m, n): A v=0\} \cap \overline{c o}(f) .
$$

Theorem 3.4. Let $U$ be a convex open subset of $\mathbf{R}^{m}$. A locally Lipschitz tame mapping $f: U \rightarrow \mathbf{R}^{n}$ is injective if it satisfies the following conditions:

$\left(C_{e}\right)$ for any $v \in \mathbf{R}^{n},|v|=1$ the set $\Sigma_{v}(f)$ is extremal in $\overline{c o}(f)$,

(S) $f$ is not constant on any segment in $U$.

Remark 3.5. Note that a priori we do not assume that there is at least one point in $U$ at which the Jacobi matrix of $f$ is invertible.

Example 3.6. Consider the homeomorphism $f: \mathbf{R}^{2} \rightarrow \mathbf{R}^{2}$ defined by $(x, y) \mapsto$ $\left(x^{3}, y^{3}\right)$. The Jacobi's matrices are given by $\left(\begin{array}{cc}3 x^{2} & 0 \\ 0 & 3 y^{2}\end{array}\right)$. We observe that $\overline{c o}(f)$ is 
the set of all matricies $\left(\begin{array}{ll}a & 0 \\ 0 & b\end{array}\right)$, where $a, b \geq 0$. For $v=( \pm 1,0)$ and $v=(0, \pm 1)$ the corresponding sets $\Sigma_{v}(f)$ are the closed halflines in the boundary of $\overline{c o}(f)$, so they are extremal in $\overline{c o}(f)$. For any other $v$ the set $\Sigma_{v}(f)$ is just the matrix $A=0$ which is extremal in $\overline{c o}(f)$. Hence the condition $\left(C_{e}\right)$ is satisfied.

It seems rather difficult to weaken the assumptions in Theorem 3.4. To see this let us consider the following examples.

Example 3.7. Consider a continuous map $f: \mathbf{R}^{2} \rightarrow \mathbf{R}^{2}$ defined by $f(x, y)=$ $\left(x+y^{3}, x\right)$ for $x \geq 0$ and $f(x, y)=\left(x+y^{3}, 0\right)$ for $x \leq 0$. Clearly this map is not injective. The Jacobi's matrices of $f$ are of the form $\left(\begin{array}{ll}1 & a \\ 1 & 0\end{array}\right), a \geq 0$ for $x \geq 0$, and $\left(\begin{array}{ll}1 & a \\ 0 & 0\end{array}\right), a \geq 0$ for $x \leq 0$. So $\overline{c o}(f)$ is the set of all matrices $\left(\begin{array}{ll}1 & a \\ b & 0\end{array}\right)$, where $a \geq 0,0 \leq b \leq 1$. Clearly $\Sigma \cap \overline{c o}(f)$ are all the matricies such that $a b=0$. Hence $\Sigma \cap \overline{c o}(f)$ does not contain any matrix from the relative interior of $\overline{c o}(f)$. It can be easily checked that, for almost all $v \in \mathbf{R}^{2}$, the set $\Sigma_{v}(f)$ is just one matrix of the form $\left(\begin{array}{ll}1 & a \\ 0 & 0\end{array}\right), a>0$ which is not an extremal point of $\overline{c o}(f)$. Hence the condition $\left(C_{e}\right)$ is not satisfied.

Example 3.8. Similarly, let $f: \mathbf{R}^{3} \rightarrow \mathbf{R}^{3}$ be an analytic function defined by $f(x, y, z)=\left(y^{3}, z y^{2}, x+z^{3}\right)$. Note that $f$ is nonsingular precisely outside $y=0$ and clearly is not constant on any segment. Moreover $\overline{c o}(f)$ has no singular matrices in its relative interior, and $f$ is obviously not injective. $A(a)=\left(\begin{array}{ccc}0 & 0 & 0 \\ 0 & 0 & 0 \\ 1 & 0 & a\end{array}\right) \in \overline{c o}(f), a \geq 0$, and we can use Proposition 2.7, with $v=(1,0,-1) \in \operatorname{ker}(A(1 / 2)+A(3 / 2))$, to contradict the extremality condition $\left(C_{e}\right)$.

Note that Theorem 3.3 holds for any continuous (possibly non-Lipschitz) function, however in Theorem 3.4 we need to assume that $f$ is locally Lipschitz. In fact we may drop this assumption but we need to strengthen the condition $\left(C_{e}\right)$. Precisely, we need to control the set $\Sigma_{v}(f)$ at "infinity". For any vector $v \in \mathbf{R}^{n},|v|=1$, we put

$$
\Sigma_{v}^{c}(f):=\{A \in M(m, n): A v=0\} \cap \overline{c o n e}(f) .
$$

Clearly $\Sigma_{v}^{c}(f)$ is a closed convex cone in the space of matrices $M(m, n)$. Now we can state our next theorem.

Theorem 3.9. Let $U$ be a convex open subset of $\mathbf{R}^{m}$. A continuous tame mapping $f: U \rightarrow \mathbf{R}^{n}$ is injective if it satisfies the following conditions:

$\left(C_{e}^{c}\right)$ for any $v \in \mathbf{R}^{n},|v|=1$ the set $\Sigma_{v}^{c}(f)$ is semi-extremal in $\overline{c o n e}(f)$,

(S) $f$ is not constant on any segment in $U$.

Remark 3.10. Actually Theorem 3.9 implies Theorem 3.4. To see this, note that for a Lipschitz function, the set $\overline{c o}(f)$ is compact and convex. It follows from Lemma 2.5 that $\overline{c o n e}(f)$ is a closed convex cone. Condition $\left(C_{e}^{c}\right)$ implies Condition $\left(C_{e}\right)$. 
We point out that the Example 3.19, a biLipschitz homeomorphism $f: \mathbf{R}^{2} \rightarrow \mathbf{R}^{2}$, does not satisfy the condition $\left(C_{e}\right)$. This motivates another generalization of the inverse mapping theorem.

Theorem 3.11. Let $f:\left(\mathbf{R}^{n}, 0\right) \rightarrow\left(\mathbf{R}^{n}, 0\right)$ be a tame continuous map-germ. Then $f$ is a homeomorphism, if there are coordinates systems $\left(x_{1}, \ldots, x_{n}\right),\left(y_{1}, \ldots, y_{n}\right)$ of the source and the target satisfying the following conditions:

$\left(R_{j}\right)$ for each $j=1, \ldots, n-1$, there are positive constants $K_{j}, L_{j}$ such that

$$
K_{j} \leq \operatorname{det} \frac{\partial\left(f_{1}, \ldots, f_{j}\right)}{\partial\left(x_{1}, \ldots, x_{j}\right)} \leq L_{j} \quad \text { except on } B(f) ;
$$

$\left(R_{n}\right)$ there is a positive constant $K_{n}$ such that

$$
K_{n} \leq \operatorname{det} \frac{\partial\left(f_{1}, \ldots, f_{n}\right)}{\partial\left(x_{1}, \ldots, x_{n}\right)} \quad \text { except on } B(f) .
$$

Remark 3.12. Note that a global version of this result is wrong. An analytic counter-exemple was given by Gale and Nikaido [4], when answering a question of Samuelson whether the strict positivity of upper-left principal minors is a sufficient condition for the univalence.

We remark that $f$ and $f^{-1}$ may not be Lipschitz, as Example 3.20 shows. We also remark that Condition $\left(R_{n}\right)$ itself is not sufficient to ensure the injectivity, as Example 3.21 shows. This theorem applies to Example 3.19, but not to Example 3.6. So different generalizations are desirable.

Theorem 3.13. A continuous tame map-germ $f:\left(\mathbf{R}^{n}, 0\right) \rightarrow\left(\mathbf{R}^{n}, 0\right)$ is a homeomorphism, if there are systems of coordinates $\left(x_{1}, \ldots, x_{n}\right),\left(y_{1}, \ldots, y_{n}\right)$ of the source and the target which satisfy the following conditions:

$\left(F_{j}\right)$ for each $j=1, \ldots, n$ the mapping $\phi_{j}:\left(\mathbf{R}^{n}, 0\right) \rightarrow\left(\mathbf{R}^{n}, 0\right)$ defined by

$$
x=\left(x_{1}, \ldots, x_{n}\right) \mapsto\left(f_{1}(x), \ldots, f_{j}(x), x_{j+1}, \ldots, x_{n}\right)
$$

is finite;

$\left(P_{j}\right)$ for each $j=1, \ldots, n$ we have $0 \leq \operatorname{det} \frac{\partial\left(f_{1}, \ldots, f_{j}\right)}{\partial\left(x_{1}, \ldots, x_{j}\right)}$ except on $B(f)$.

We remark that Conditions $\left(F_{j}\right)$ cannot be dropped in Theorem 3.13, as we see in Example 3.14.

Example 3.14. Consider the mapping $f:\left(\mathbf{R}^{2}, 0\right) \rightarrow\left(\mathbf{R}^{2}, 0\right)$ defined by

$$
(x, y) \mapsto\left(x, x^{2} y\right) .
$$

Then the Jacobi's matrix of $f$ is

$$
d f(x, y)=\left(\begin{array}{cc}
1 & 0 \\
2 x y & y^{2}
\end{array}\right) .
$$

The Condition $\left(C_{e}\right)$ is satisfied. Indeed, observe that $\overline{c o}(f)$ is the set of all matrices $\left(\begin{array}{ll}1 & 0 \\ a & b\end{array}\right)$, where $a \in \mathbf{R}, b \geq 0$. For $v=(0, \pm 1)$ the corresponding set $\Sigma_{v}(f)$ is the line in the boundary of $\overline{c o}(f)$, so it is extremal in $\overline{c o}(f)$. For any other $v$ the set $\Sigma_{v}(f)$ is empty so is extremal in $\overline{c o}(f)$. However, Condition $(\mathrm{S})$ is not satisfied, since the image of $x$-axis is just the origin. Obviously the map $f$ is not a homeomorphism. 
Note that Condition $\left(R_{1}\right)$ is satisfied but not Condition $\left(R_{2}\right)$. Also note that Conditions $\left(F_{1}\right),\left(P_{1}\right),\left(P_{2}\right)$ are satisfied, but Condition $\left(F_{2}\right)$ is not satisfied.

Example 3.15. Let $U$ be a convex open neighbourhood of 0 in $\mathbf{R}^{2}$. Consider the mapping $f: U \rightarrow \mathbf{R}^{2}$ defined by

$$
(x, y) \mapsto\left(x\left(y-x^{2}\right)^{2}, y-x^{2}\right) .
$$

The Jacobi matrix of $f$ is

$$
d f(x, y)=\left(\begin{array}{ll}
a_{11} & a_{12} \\
a_{21} & a_{22}
\end{array}\right)=\left(\begin{array}{cc}
\left(x^{2}-y\right)\left(5 x^{2}-y\right) & 2 x\left(y-x^{2}\right) \\
-2 x & 1
\end{array}\right) .
$$

We show that $\operatorname{ri}(c o(f))$ contains singular matrices. Since $a_{11}=\left(x^{2}-y\right)\left(5 x^{2}-y\right)$ changes its sign, it is enough to show that the relative interior of $c o(f) \cap\left\{a_{11}=0\right\}$ contains singular matrices, matrices with $a_{12} a_{21}=0$. Since

$$
\mathcal{D}(f) \cap\left\{a_{11}=0\right\}=\left\{\left(\begin{array}{cc}
0 & 0 \\
-2 x & 1
\end{array}\right):\left(x, x^{2}\right) \in U\right\} \cup\left\{\left(\begin{array}{cc}
0 & 8 x^{3} \\
-2 x & 1
\end{array}\right):\left(x, 5 x^{2}\right) \in U\right\},
$$

the relative interior of its convex hull contains singular matrices. This is clearly not a homeomorphism, the image of $\left\{y=x^{2}\right\}$ is just the origin.

Example 3.16. Consider the mapping $f: \mathbf{R}^{2} \rightarrow \mathbf{R}^{2}$ defined by

$$
(x, y) \mapsto\left(y_{1}, y_{2}\right)=\left(x y^{2 / 3}, y^{1 / 3}\right) .
$$

Its Jacobi's matrix is

$$
\left(\begin{array}{cc}
y^{2 / 3} & \frac{2}{3} x y^{-1 / 3} \\
0 & \frac{1}{3} y^{-2 / 3}
\end{array}\right)
$$

and we have

$$
\frac{\partial y_{1}}{\partial x}=y^{2 / 3} \geq 0, \quad \frac{\partial\left(y_{1}, y_{2}\right)}{\partial(x, y)}=\frac{1}{3}>0 .
$$

Note the image of $x$-axis is the origin hence $f$ is not injective. We remark that this example satisfies Conditions $\left(C_{e}\right),\left(R_{2}\right),\left(P_{1}\right),\left(P_{2}\right)$, but not Conditions $(\mathrm{S}),\left(R_{1}\right)$ and $\left(F_{2}\right)$.

Example 3.17. Consider the mapping $f: \mathbf{R}^{2} \rightarrow \mathbf{R}^{2}$ defined by

$$
(x, y) \mapsto\left(y_{1}, y_{2}\right)=\left(x y^{1 / 3}, y^{2 / 3}\right) .
$$

The Jacobi's matrix is

$$
\left(\begin{array}{cc}
y^{1 / 3} & \frac{1}{3} x y^{-2 / 3} \\
0 & \frac{2}{3} y^{-1 / 3}
\end{array}\right)
$$

and its Jacobian determinant is $2 / 3$. Note the image of $f$ is $\left\{y_{2}>0\right\} \cup\{(0,0)\}$, which is not dense. We remark that this example satisfies Conditions $\left(C_{e}\right),\left(R_{2}\right)$, $\left(P_{1}\right),\left(P_{2}\right)$, but not Conditions $(\mathrm{S})$ and $\left(R_{1}\right),\left(F_{2}\right)$.

Let $P: \mathbf{R}^{n}-\{0\} \rightarrow(0, \infty)$ denote a function satisfying

$$
P\left(t^{w_{1}} x_{1}, \ldots, t^{w_{n}} x_{n}\right)=t^{d} P\left(x_{1}, \ldots, x_{n}\right), \quad t \in(0, \infty)
$$

where $w_{1}, \ldots, w_{n}, d$ are real numbers. Let $F_{P}: \mathbf{R}^{n}-\{0\} \rightarrow \mathbf{R}^{n}-\{0\}$ be the mapping defined by

$$
F_{P}\left(x_{1}, \ldots, x_{n}\right)=\left(P(x)^{w_{1}} x_{1}, \ldots, P(x)^{w_{n}} x_{n}\right) .
$$

If $(d+1)\left(d^{\prime}+1\right)=1$ define $Q(x)$ by $Q(x) P^{\left(d^{\prime}+1\right)}(x) \equiv 1$ and accordingly the corresponding map $F_{Q}$. 
Proposition 3.18. $F_{P}^{-1}=F_{Q}$, in particular $F_{P}$ is a homeomorphism whenever $P$ is continuous. If moreover $(d+1)>0, w_{i}>0, i=1, \ldots, n$, then $F_{P}$ extends to a global homeomorphism of $\mathbf{R}^{n}$.

Proof. This follows from the following computation:

$$
\begin{aligned}
F_{Q^{\circ}} F_{P}(x) & =F_{Q}\left(P(x)^{w_{1}} x_{1}, \ldots, P(x)^{w_{n}} x_{n}\right) \\
& \left.=\left(Q\left(P(x)^{w_{1}} x_{1}, \ldots, P(x)^{w_{n}} x_{n}\right)\right)^{w_{i}}\left(P(x)^{w_{i}} x_{i}\right)\right)_{i=1, \ldots, n} \\
& =\left(\left(P(x)^{d^{\prime}} Q(x)\right)^{w_{i}} P(x)^{w_{i}} x_{i}\right)_{i=1, \ldots, n} \\
& =\left(P(x)^{\left(d^{\prime}+1\right) w_{i}} Q(x)^{w_{i}} x_{i}\right)_{i=1, \ldots, n} \\
& =\left(x_{i}\right)_{i=1, \ldots, n} .
\end{aligned}
$$

Finally observe that $\lim _{x \rightarrow 0} F_{P}=0$ if $d w_{i}+w_{i}>0$ for all $i=1, \ldots, n$.

Example 3.19. Consider the mapping $f:\left(\mathbf{R}^{2}, 0\right) \rightarrow\left(\mathbf{R}^{2}, 0\right)$ defined by

$$
f(x, y)=\left\{\begin{array}{ll}
(P x, P y) & (x, y) \neq(0,0) \\
(0,0) & (x, y)=(0,0)
\end{array} \quad \text { where } \quad P=\frac{x^{4}-x^{2} y^{2}+2 y^{4}}{x^{4}-x^{2} y^{2}+y^{4}}\right.
$$

By Proposition 3.18, we see that this is a homeomorphism. Remark that the inverse is given by

$$
(x, y) \mapsto \begin{cases}\left(P^{-1} x, P^{-1} y\right) & (x, y) \neq(0,0) \\ (0,0) & (x, y)=(0,0)\end{cases}
$$

The Jacobi's matrix of $f$ is

$$
\left(\begin{array}{ll}
a_{11} & a_{12} \\
a_{21} & a_{22}
\end{array}\right)=\frac{1}{\left(x^{4}-x^{2} y^{2}+y^{4}\right)^{2}}\left(\begin{array}{cc}
\left(x^{2}-2 y^{2}\right)\left(x^{6}-y^{6}\right) & 2 x^{3} y^{3}\left(2 x^{2}-y^{2}\right) \\
2 x y^{5}\left(y^{2}-2 x^{2}\right) & x^{8}-2 x^{6} y^{2}+8 x^{4} y^{4}-5 x^{2} y^{6}+2 y^{8}
\end{array}\right),
$$

and we observe each entry is bounded. Hence $f$ is Lipschitz. Similarly we can show $f^{-1}$ is Lipschitz, so $f$ is a biLipschitz homeomorphism. We observe that

$$
\frac{\partial f_{2}}{\partial y}=\frac{\left(x^{2}-y^{2}\right)^{4}+y^{4}\left(x^{2}-y^{2}\right)^{2}+x^{2} y^{2}\left(2 x^{4}+x^{2} y^{2}+y^{4}\right)}{\left(x^{4}-x^{2} y^{2}+y^{4}\right)^{2}} \geq 1, \text { and } \operatorname{det}(d f)=P^{2} \geq 1 .
$$

We use Theorem 3.11 to show that $f$ is a homeomorphism germ. Consider the segment

$$
\gamma_{\varepsilon}:[-2 \varepsilon, 2 \varepsilon] \rightarrow \mathbf{R}^{2}, \quad t \mapsto(t, \pm \varepsilon) \quad \text { for } 0<\varepsilon \ll 1 .
$$

The images of $f \circ \gamma_{\varepsilon}$ look like the following:
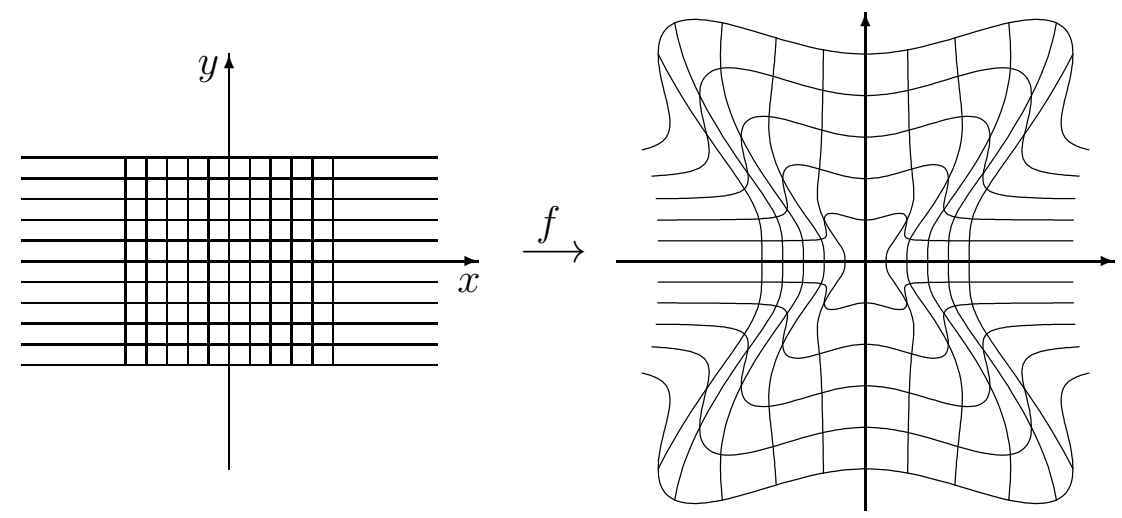
We now show that $\operatorname{ri}(c o(f))$ contains singular matrices, hence in particular $f$ does not satisfy the condition $\left(C_{e}\right)$. First one can see that the affine hull of $c o(f)$ is $M(2,2)$. Indeed the following matrices from $c o(f)$ are affine independent

$$
\left(\begin{array}{ll}
1 & 0 \\
0 & 1
\end{array}\right), \quad\left(\begin{array}{cc}
0 & -2 \\
2 & 4
\end{array}\right), \quad\left(\begin{array}{cc}
0 & 2 \\
-2 & 4
\end{array}\right), \quad\left(\begin{array}{cc}
0 & -\frac{2 \sqrt{2}}{3} \\
\frac{4 \sqrt{2}}{3} & \frac{8}{3}
\end{array}\right), \quad\left(\begin{array}{cc}
0 & \frac{2 \sqrt{2}}{3} \\
-\frac{4 \sqrt{2}}{3} & \frac{8}{3}
\end{array}\right)
$$

Next we observe that $a_{11}=\frac{\left(x^{2}-2 y^{2}\right)\left(x^{6}-y^{6}\right)}{\left(x^{4}-x^{2} y^{2}+y^{4}\right)^{2}}$ changes its sign. So it is enough to show that the relative interior of $c o(f) \cap\left\{a_{11}=0\right\}$ contains a singular matrix. If $a_{11}=0$, then $y= \pm x, \pm x / \sqrt{2}$, and we observe that the last four matrices from the list above are realized as Jacobi's matrices precisely in $\left\{a_{11}=0\right\}$. Since the singular matrices in the space $\left\{a_{11}=0\right\}$ satisfy $\left\{a_{12} a_{21}=0\right\}$, the relative interior of the convex hull of those four matrices contains singular matrices. This implies that ri $(c o(f))$ contains singular matrices.

This example shows that Condition $\left(C_{e}\right)$ is not invariant by biLipschitz mappings.

Example 3.20. Let $f:\left(\mathbf{R}^{2}, 0\right) \rightarrow\left(\mathbf{R}^{2}, 0\right)$ be a mapping defined by

$$
f(x, y)=\left\{\begin{array}{ll}
\left(P^{3} x, P^{2} y\right) & (x, y) \neq(0,0) \\
(0,0) & (x, y)=(0,0)
\end{array} \text { where } P=\frac{2 x^{4}+y^{6}}{x^{4}+y^{6}}\right.
$$

By Proposition 3.18, we see that this is a homeomorphism. We obtain that

$$
d f=\frac{\left(2 x^{4}+y^{6}\right)}{\left(x^{4}+y^{6}\right)^{4}}\left(\begin{array}{cc}
\left(2 x^{4}+y^{6}\right)\left(2 x^{8}+15 x^{4} y^{6}+y^{12}\right) & -18 x^{5} y^{5}\left(2 x^{4}+y^{6}\right) \\
8 x^{3} y^{7}\left(x^{4}+y^{6}\right) & \left(x^{4}+y^{6}\right)\left(2 x^{8}-9 x^{4} y^{6}+y^{12}\right)
\end{array}\right),
$$

and observe that all components but $\frac{\partial f_{2}}{\partial x}$ are bounded, so this mapping is not Lipschitz. Moreover, we observe that

$$
\frac{\partial f_{1}}{\partial x}=\frac{\left(2 x^{4}+y^{6}\right)\left(2 x^{8}+15 x^{4} y^{6}+y^{12}\right)}{\left(x^{4}+y^{6}\right)} \geq 1, \text { and } \operatorname{det}(d f)=P^{5} \geq 1 .
$$

So we can use Theorem 3.11 also to show that $f$ is a homeomorphism germ. Consider the segment

$$
\gamma_{x_{0}}:[-\varepsilon, \varepsilon] \rightarrow \mathbf{R}^{2}, \quad t \mapsto\left(x_{0}, t\right) \quad \text { for } \quad 0<\varepsilon \ll\left|x_{0}\right| \ll 1 .
$$

Then we have

$$
d f\left(\gamma_{x_{0}}(t)\right) \gamma_{x_{0}}^{\prime}(t)=\frac{\left(2 x_{0}^{4}+t^{6}\right)}{\left(x_{0}^{4}+t^{6}\right)^{4}}\left(-18 x_{0}^{5} t^{5}\left(2 x_{0}^{4}+t^{6}\right),\left(x_{0}^{4}+t^{6}\right)\left(2 x_{0}^{8}-9 x_{0}^{4} t^{6}+t^{12}\right)\right)
$$

and we observe that the first component changes the sign at $t=0$ and the second component changes the sign four times. Thus the images of $f \circ \gamma_{x_{0}}$ look like the following: 

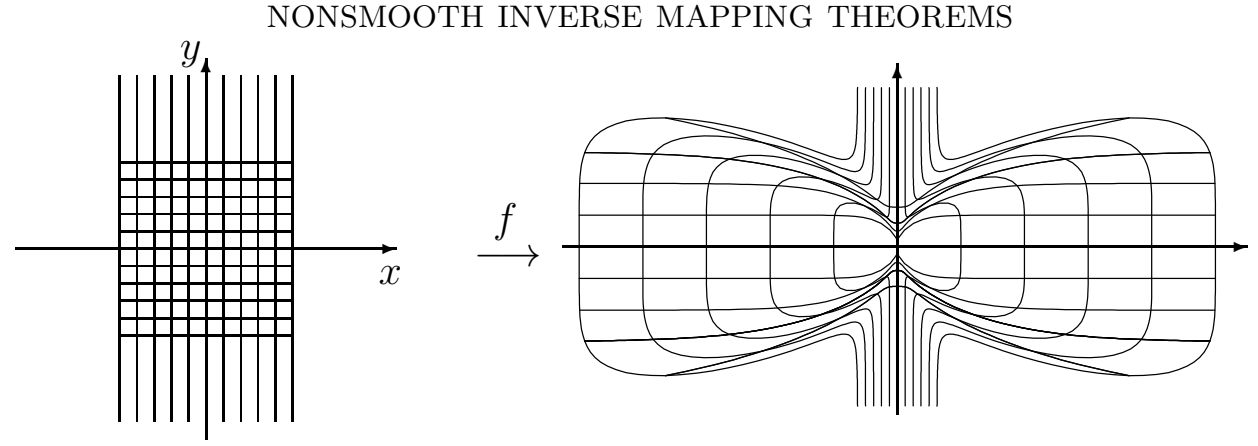

We see that $\operatorname{ri}(\operatorname{co}(f))$ contains singular matrices.

Example 3.21. Let $f:\left(\mathbf{R}^{2}, 0\right) \rightarrow\left(\mathbf{R}^{2}, 0\right)$ be a mapping defined by

$$
f(x, y)= \begin{cases}\frac{1}{x^{2}+y^{2}}\left(x\left(x^{2}-3 y^{2}\right), y\left(3 x^{2}-y^{2}\right)\right) & (x, y) \neq(0,0) \\ (0,0) & (x, y)=(0,0)\end{cases}
$$

Then we have

$$
d f=\frac{1}{\left(x^{2}+y^{2}\right)^{2}}\left(\begin{array}{cc}
x^{4}+6 x^{2} y^{2}-3 y^{4} & 8 x y^{3} \\
-8 x^{3} y & 3 x^{4}-6 x^{2} y^{2}-y^{4}
\end{array}\right)
$$

and $\operatorname{det}(d f)=3$. We then obtain that

$$
\begin{aligned}
& \frac{1}{3} d f(r, 0)+\frac{1}{3} d f\left(r \cos \frac{2 \pi}{3}, r \sin \frac{2 \pi}{3}\right)+\frac{1}{3} d f\left(r \cos \frac{2 \pi}{3},-r \sin \frac{2 \pi}{3}\right) \\
& =\frac{1}{3}\left(\begin{array}{ll}
1 & 0 \\
0 & 3
\end{array}\right)+\frac{1}{3}\left(\begin{array}{cc}
-\frac{1}{2} & \frac{\sqrt{3}}{2} \\
-\frac{3 \sqrt{3}}{2} & -\frac{3}{2}
\end{array}\right)+\frac{1}{3}\left(\begin{array}{cc}
-\frac{1}{2} & -\frac{\sqrt{3}}{2} \\
\frac{3 \sqrt{3}}{2} & -\frac{3}{2}
\end{array}\right)=\left(\begin{array}{ll}
0 & 0 \\
0 & 0
\end{array}\right),
\end{aligned}
$$

and so ri $(c o(f))$ contains a singular matrix. We see that

$$
f(r \cos \theta, r \sin \theta)=(r \cos 3 \theta, r \sin 3 \theta)
$$

and that the mapping $f$ is a 3 -sheeted branched covering at 0 .

\section{O-minimal key lemma}

The purpose of this section is to show the key Lemma 4.3. It will allow us to extend our control on directional derivatives from an open dense set to the whole domain of the considered map.

Definition 4.1. An o-minimal structure on $(\mathbf{R},+, \cdot,<)$ (cf. [1] or [13]) is a sequence of boolean algebras $\mathcal{O}_{n}$ of definable subsets of $\mathbf{R}^{n}$, such that for each $n \in \mathbf{N}$

- if $A \in \mathcal{O}_{m}$ and $B \in \mathcal{O}_{n}$, then $A \times B \in \mathcal{O}_{m+n}$;

- if $\Pi: \mathbf{R}^{n+1} \rightarrow \mathbf{R}^{n}$ is the canonical projection onto $\mathbf{R}^{n}$ then for any $A \in \mathcal{O}_{n+1}$, the set $\Pi(A)$ belongs to $\mathcal{O}_{n}$;

- $\mathcal{O}_{n}$ contains the family of algebraic subsets of $\mathbf{R}^{n}$, that is, every set of the form $\left\{x \in \mathbf{R}^{n}: p(x)=0\right\}$, where $p: \mathbf{R}^{n} \rightarrow \mathbf{R}$ is a polynomial function;

- the elements of $\mathcal{O}_{1}$ are exactly the finite unions of intervals and points. 
Definition 4.2. Given an o-minimal structure $\mathcal{O}$ on $(\mathbf{R},+, \cdot,<)$ and $U \subset \mathbf{R}^{m}$, we say that a mapping $U \rightarrow \mathbf{R}^{n} \cup\{+\infty\}$ is definable in $\mathcal{O}$ if its graph belongs to $\mathcal{O}_{m+n}$. We say for short that $f$ is definable if it is definable in some o-minimal structure $\mathcal{O}$ on $(\mathbf{R},+, \cdot,<)$.

Let $U$ be an open set of $\mathbf{R}^{m}$ and let $f: U \rightarrow \mathbf{R}^{n}$ be a continuous definable mapping. Recall that the set $B(f)$ of the points where $f$ is not differentiable, is a nowhere dense subset of $\mathbf{R}^{m}$. If $A=d f(x) \in \mathcal{D}(f)$, then $A v=\partial_{v} f(x) \in \mathcal{D}_{v}(f)$, the directional derivative of $f$ at $x$ in the direction $v$.

We state now the key lemma.

Lemma 4.3. Let $U$ be an open subset of $\mathbf{R}^{m}$ and let $f: U \rightarrow \mathbf{R}^{n}$ be a continuous definable mapping. Fix $x \in U$ and $v \in \mathbf{R}^{m},|v|=1$. Let $g(t)=f(x+t v), t \in[a, b]$. Then $g^{\prime}(t) \in \overline{\mathcal{D}_{v}(f)}$, whenever $g^{\prime}(t)$ exists.

Proof. Clearly it is enough to consider the case $n=1$. Since $f$ is a continuous definable mapping, $g$ is a continuous definable function. Hence, see [1] or [13], $g^{\prime}(t)$ exists (and is continuous) except for finitely many points. It is thus enough to show the lemma for a generic $t$, that is, for all but finitely many $t \in[a, b]$. So, in the proof we shall replace the segment $[a, b]$ by a subsegment, but for simplicity we shall denote it again by $[a, b]$. First we explain the reduction to the case $m=2$. Let $S_{v}$ denote the unit sphere in the orthogonal complement of $v$. For any $u \in S_{v}$ we define a map

$$
p_{u, v}:[a, b] \times[0,+\infty) \ni(t, s) \mapsto x+t v+s u \in \mathbf{R}^{m} .
$$

Lemma 4.4. There exists $u \in S_{v}$ such that $\operatorname{dim} p_{u, v}^{-1}(B(f))=1$. Actually the set of all such $u$ is dense in $S_{v}$.

Proof. Assume that there exists an open non-empty set $W \subset S_{v}$ such that the corresponding sets $p_{u, v}^{-1}(B(f)), u \in W$, are of dimension 2. We have a natural projection

$$
\pi: \mathbf{R}^{m} \backslash \mathbf{R} v \rightarrow S_{v},
$$

which is a composition of: the translation by $-x$, the orthogonal projection on the orthogonal complement of $v$ and next the radial projection on $S_{v}$. Note that for any $u \in S_{v}$, the image of $p_{u, v}$ is contained in the fiber $\pi^{-1}(u)$. It follows from the formula for dimension of a definable set that

$$
\operatorname{dim} B(f) \geq \min \left\{\operatorname{dim} \pi^{-1}(u) \cap B(f), u \in W\right\}+\operatorname{dim} W=2+(m-2)=m,
$$

which is a contradiction, since $B(f)$ is a nowhere dense set, so $\operatorname{dim} B(f)<m$.

To achieve the proof of Lemma 4.3 we fix $u \in S_{v}$ such that $\operatorname{dim} p_{u, v}^{-1}(B(f))=1$. Then, for some $\varepsilon>0$ small enough, we consider the following continuous definable function

$$
F(t, s)=f\left(x_{0}+t v+s u\right)-f\left(x_{0}+t v\right), t \in[a, b], s \in[0, \varepsilon) .
$$

Clearly $F(t, 0)=0$. Since $\operatorname{dim} p_{u, v}^{-1}(B(f))=1$, applying the cell decomposition (c.f. [1] or [13]) to $p_{u, v}^{-1}(B(f))$ we may assume (changing suitably $a$ and $b$ ) that $F$ is differentiable (even 2-times differentiable) in $[a, b] \times(0, \varepsilon)$. Moreover $\frac{\partial F}{\partial t}(t, s)=$ 
$\partial_{v} f\left(p_{u, v}(t, s)\right)-g^{\prime}(t)$. So it is enough to show $\lim _{s \rightarrow 0} \frac{\partial F}{\partial t}(t, s)=0$, for generic $t$. Consider the definable set

$$
Z=\left\{(t, s) \in\left(t^{*}-\delta, t^{*}+\delta\right) \times(0, \varepsilon): \frac{\partial F}{\partial t}(t, s)=0, \frac{\partial^{2} F}{\partial t^{2}}(t, s)=0\right\} .
$$

Applying the cell decomposition to $Z$ we may assume that $\frac{\partial F}{\partial t}(t, s)$ and $\frac{\partial^{2} F}{\partial t^{2}}(t, s)$ are of constant sign on $[a, b] \times(0, \varepsilon)$. Let us assume that both partials are strictly positive (the other cases are similar).

We claim that, for generic $t, \frac{\partial F}{\partial t}(t, s) \rightarrow 0$ as $s \rightarrow 0$. Assume that for some $t^{*} \in[a, b)$ we have $\frac{\partial F}{\partial t}\left(t^{*}, s\right) \rightarrow 2 c>0$, as $s \rightarrow 0$. (A priori we may have $c=+\infty$, we leave to the reader to adapt the argument below to this case.) For each $s \in(0, \varepsilon)$ the function $t \rightarrow \frac{\partial F}{\partial t}(t, s)$ is increasing, so for any $s>0$ small enough we have

$$
\frac{\partial F}{\partial t}(t, s) \geq c, t \in\left[t^{*}, b\right] .
$$

By the Mean Value Theorem it follows that

$$
F(t, s) \geq F\left(t^{*}, s\right)+c\left(t-t^{*}\right), t \in\left[t^{*}, b\right] .
$$

So taking limit as $s \rightarrow 0$, we obtain

$$
F(t, 0) \geq F\left(t^{*}, 0\right)+c\left(t-t^{*}\right), t \in\left[t^{*}, b\right] .
$$

But this is absurd since $F(t, 0)=0, t \in\left[t^{*}, b\right]$.

4.1. Tame, subanalytic and definable mappings. We explain below the notion of tame mapping we use in this paper.

Definition 4.5. $X \subset \mathbf{R}^{n}$ is semianalytic if, for all $x \in \mathbf{R}^{n}$, there is an open neighborhood $U$ of $x$ such that $X \cap U$ is a finite Boolean combination of sets $\{x \in$ $U: f(x)=0\}$ and $\{x \in U: g(x)>0\}$, where $f, g: U \rightarrow \mathbf{R}$ are analytic.

Definition 4.6. $X \subset \mathbf{R}^{n}$ is subanalytic (cf. [10], [11]) if, for all $x \in \mathbf{R}^{n}$, there is an open set $U$ and a bounded semianalytic set $Y \subset \mathbf{R}^{n+m}$ such that $X \cap U$ is the projection of $Y$ into $U$.

Let us recall that the collection of global subanalytic sets form an o-minimal structure. (Recall that $A \subset \mathbf{R}^{n}$ is globally subanalytic if $A$ is subanalytic as subset of the projective space $\mathbf{P}^{n}$, for the natural embedding $\left.\mathbf{R}^{n} \rightarrow \mathbf{P}^{n}\right)$. In particular, if $U \subset \mathbf{R}^{n}$ is bounded and $f: U \rightarrow \mathbf{R}$ is a bounded subanalytic function then $f$ is definable, that is, $f$ is globally subanalytic. Both conditions are actually necessary.

Recall that we call a continuous map $f: U \rightarrow \mathbf{R}^{n}$, where $U \subset \mathbf{R}^{m}$ is open, tame if its graph is definable in an o-minimal structure or subanalytic. Note that Lemma 4.3 applies also to subanalytic functions since we may assume that $f$ is bounded.

4.2. Directional derivatives of tame mappings. Let $v \in S^{m-1}$. Recall that

$$
\partial_{v} f(x)=\lim _{h \rightarrow+0} \frac{f(x+h v)-f(x)}{h} .
$$

is the directional derivative of $f$ at $x$ in direction $v$. Let $f: U \rightarrow \mathbf{R}^{n}$ be a tame function, where $U$ is an open subset of $\mathbf{R}^{n}$. 
Let $B_{v}(f)$ be the set of points $x \in U$ such that $\partial_{v} f(x)$ does not exists (precisely equals $+\infty$ or $-\infty)$. Recall that $B_{v}(f) \subset B(f)$, where $B(f)$ is the set of points at which $f$ is not differentiable. We denote

$$
\operatorname{Dir}_{v}(f)=\left\{\partial_{v} f(x): x \in U \backslash B_{v}(f)\right\},
$$

and

$$
\overline{c o}_{v}(f)=\overline{c o}\left(\operatorname{Dir}_{v}(f)\right) \text {. }
$$

Recall that $\mathcal{D}_{v}(f)=\{A v: A \in \mathcal{D}(f)\}$, where $\mathcal{D}(f)=\{d f(x): x \in U-B(f)\}$. Note that Lemma 4.3 implies the following statement.

Proposition 4.7. Let $U$ be an open subset of $\mathbf{R}^{n}$ and $f: U \rightarrow \mathbf{R}^{n}$ a continuous mapping which is tame (i.e subanalytic or definable in an o-minimal structure). Then $\overline{\operatorname{Dir}_{v}(f)}=\overline{\mathcal{D}_{v}(f)}$, hence

$$
\overline{c o}_{v}(f)=\overline{c o}\left(\mathcal{D}_{v}(f)\right) .
$$

Let us consider the linear map $\varphi: M(m, n) \rightarrow \mathbf{R}^{n}, \varphi(A)=A v$. We know by Proposition 4.7 that $\overline{c o}_{v}(f)=\overline{c o}(\varphi(c o(f))$. Let us assume now that $f$ is Lipschitz, hence $\overline{c o}(f)$ is compact. Thus

$$
\overline{c o}_{v}(f)=\overline{c o}(\varphi(\overline{c o}(f))=\varphi(\overline{c o}(f)) .
$$

This shows that the condition $\left(C_{e}\right)$ and Lemma 2.4 imply the following important fact.

Lemma 4.8. If $f$ is Lipschitz and satisfies condition $\left(C_{e}\right)$, then the vector $0 \in \mathbf{R}^{n}$ is an extremal point in $\overline{c o}_{v}(f)$.

Similarly, for the need of the proof of Theorem [3.9, we consider closed convex cones. We put $\overline{\operatorname{cone}}_{v}(f):=\overline{c o n e}\left(\mathcal{D}_{v}(f)\right)$. Then Proposition 4.7 and Lemma 2.6 yields

$$
\overline{\operatorname{cone}}_{v}(f)=\varphi(\overline{\operatorname{cone}}(f)),
$$

since $\varphi(\overline{c o n e}(f))$ is a closed convex cone.

Note that $\overline{c o}_{v}(f) \subset \overline{\operatorname{cone}}_{v}(f)$, so we conclude from the above discussion that the condition $\left(C_{e}^{c}\right)$ and Lemma 2.4 imply the following important fact.

Lemma 4.9. If $f$ is continous and satisfies condition $\left(C_{e}^{c}\right)$, then the vector $0 \in \mathbf{R}^{n}$ is an extremal point in $\overline{c o n e}_{v}(f)$. Hence, also $0 \in \mathbf{R}^{n}$ is an extremal point in $\overline{c o}_{v}(f)$.

\section{Proof of Theorems [3.2, 3.3, 3.4.}

Lemma 5.1. Take $A \in M(m, n)$ and $v \in S^{m-1}$. If $\operatorname{dist}(A, \Sigma) \geq \delta$, then $|A v| \geq \delta$.

Proof. By Lemma 2.8, we have $\operatorname{dist}(A, \Sigma)=\inf \left\{|A v|: v \in S^{m-1}\right\}$, so the lemma is clear.

Next we state a classical lemma on the projection on a convex closed subset.

Lemma 5.2. Let $C \subset \mathbf{R}^{n}$ be a convex and closed subset with $0 \notin C$. Then there exists unique $w \in C$ such that

- $|w|=\inf \{|u|: u \in C\}$.

- if $x \in C$ then $\left\langle\frac{w}{|w|}, x\right\rangle \geq|w|>0$. 
Proof of Theorem 3.2. Take $x, x^{\prime} \in U, x \neq x^{\prime}$ and put $v=\frac{x^{\prime}-x}{\left|x^{\prime}-x\right|}, v \in S^{m-1}$. We consider the set

$$
c o_{v}(f)=\{A v: A \in c o(f)\} .
$$

By Lemma 5.1, if $u \in \overline{c o_{v}(f)}$ then $|u| \geq \delta$. So Lemma 5.2 implies that there exists a unique $w \in \overline{c O_{v}(f)}$ such that

$$
|w|=\inf \left\{|x|: x \in c o_{v}(f)\right\} .
$$

Note that by Lemma 5.1, we have $|w| \geq \delta$. Hence Lemma 5.2 yields

$$
\left\langle\frac{w}{|w|}, u\right\rangle \geq \delta \quad \text { if } u \in \operatorname{cov}_{v}(f) .
$$

Finally consider the function

$$
h(t)=\left\langle\frac{w}{|w|}, f(x+t v)-f(x)\right\rangle, \quad t \in\left[0,\left|x^{\prime}-x\right|\right] .
$$

Moving slightly $x$ and $x^{\prime}$, we may assume that, for almost all (in fact, all but finite, since $f$ is tame) $t \in\left[0,\left|x^{\prime}-x\right|\right]$, the function $f$ is differentiable at $x+t v$. Since $h^{\prime}(t)=\left\langle\frac{w}{|w|}, d f(x+t v) v\right\rangle \geq \delta$, we obtain that

$$
h\left(\left|x^{\prime}-x\right|\right)=\int_{0}^{\left|x^{\prime}-x\right|} h^{\prime}(t) d t \geq \delta\left|x^{\prime}-x\right| .
$$

So we have proved that $\left|f\left(x^{\prime}\right)-f(x)\right| \geq h\left(\left|x^{\prime}-x\right|\right) \geq \delta\left|x^{\prime}-x\right|$. Note that, if $m=n$ then $f: U \rightarrow f(U)$ is open by the invariance of domain (Lemma 2.10), so indeed $f: U \rightarrow f(U)$ is a homeomorphism.

We recall now the classical result of Minkowski about supporting hyperplanes, see e.g. 9].

Lemma 5.3. Let $C \subset \mathbf{R}^{n}$ be a convex set, and assume that relative interior of $C$ does not contain 0 . Then there exists $w \in \mathbf{R}^{n},|w|=1$ such that $\langle w, x\rangle \geq 0$, for all $x \in C$.

For the proof of the next two theorems the following lemma is crucial.

Lemma 5.4. Let $g:[a, b] \rightarrow \mathbf{R}^{n}$, $a<b$, be a tame continuous function. If the relative interior of $c o(g)$ does not contain 0 , then there is $w \in \mathbf{R}^{n}$ such that $\langle w, g(a)\rangle\langle\langle w, g(b)\rangle$. This obviously implies that $g(a) \neq g(b)$.

Proof. We show this lemma by induction on $n$. Assume $n=1$. Since the relative interior of $c o(g)$ does not contain 0 , it follows that either $g^{\prime}(t) \geq 0$ for all but finitely many $t \in[a, b]$ or $g^{\prime}(t) \leq 0$ for all but finitely many $t \in[a, b]$. Hence $g$ is monotonic. Note that $g$ is non constant, since otherwise $c o(g)=\{0\}$. Therefore $g(a) \neq g(b)$.

Assume now that $n>1$. Since 0 is not in the relative interior of $c o(g)$, by Lemma 5.3, there exists $w \in \mathbf{R}^{n},|w|=1$ such that $\left\langle w, g^{\prime}(t)\right\rangle \geq 0$. If $\left\langle w, g^{\prime}(t)\right\rangle=0$, for all but finitely many $t \in[a, b]$, then $g(t) \in H, t \in[a, b]$, for some affine hyperplane $H$ orthogonal to $w$. Hence we can apply the induction hypothesis. Otherwise the affine hull of $c o(g)$ is $\mathbf{R}^{n}$, and $\left\langle w, g^{\prime}(t)\right\rangle$ is strictly positive on an open interval. This implies

$$
\langle w, g(b)\rangle-\langle w, g(a)\rangle=\int_{a}^{b}\left\langle w, g^{\prime}(t)\right\rangle d t>0
$$


Proof of Theorem 3.3. First let us observe that by the classical local inverse theorem and by the invariance of domain, we deduce that $f$ is open on $U \backslash B$. Let us fix $x, x^{\prime} \in U, x \neq x^{\prime}$. Of course, if $x, x^{\prime} \in B$ then, by the condition $(I)$, we have $f(x) \neq f\left(x^{\prime}\right)$.

Let us assume now that $x \in U \backslash B$ and that $f(x)=f\left(x^{\prime}\right)$. Since $f$ is open at $x$, there exists $V$ an open neighborhood of $x$ such that $f(V)$ is an open neighborhood of $f(x)$. By the continuity of $f$ at $x^{\prime}$ there exists $V^{\prime}$ an open neighborhood of $x^{\prime}$ such that $V \cap V^{\prime}=\emptyset, f\left(V^{\prime}\right) \subset f(V)$. Recall that $B$ is closed and nowhere dense in $U$ hence also in $V$. So $W^{\prime}=V^{\prime} \backslash B \neq \emptyset$ is open. Now let $W=V \cap f^{-1}\left(f\left(W^{\prime}\right)\right)$. Note that for any $x^{\prime} \in W^{\prime}$ there exists $x \in W$, hence $x \neq x^{\prime}$, such that $f(x)=f\left(x^{\prime}\right)$. So we can actually assume that both $x, x^{\prime} \in U \backslash B$. Now applying Lemma 2.11 we can also suppose that $\left[x, x^{\prime}\right] \cap B$ is finite.

Let us put $v=\frac{x^{\prime}-x}{\left|x^{\prime}-x\right|}$. Set $g(t)=f(x+t v), t \in\left[0,\left|x^{\prime}-x\right|\right]$. Let us consider the linear map $\varphi: M(m, n) \rightarrow \mathbf{R}^{n}, \varphi(A)=A v$. Then $c o_{v}(f):=\varphi(c o(f, U \backslash B))$ is a convex subset of $\mathbf{R}^{n}$ which does not contain 0 . Note that

$$
g^{\prime}(t)=\partial_{v} f(x+t v)
$$

at any point $t \in\left[0,\left|x^{\prime}-x\right|\right] \backslash F$, where $F$ is a finite set. So we have

$$
\operatorname{co}(g) \subseteq \operatorname{co}_{v}(f) .
$$

We know that $0 \notin c o_{v}(f)$, hence $0 \notin c o(g)$, where $c o(g)$ is precisely the convex hull $\left\{g^{\prime}(t): t \in[a, b] \backslash F\right\}$. Thus, applying Lemma 5.4 we obtain Theorem 3.3.

Proof of Theorem 3.4. Let us fix $x, x^{\prime} \in U, x \neq x^{\prime}$ and put $v=\frac{x^{\prime}-x}{\left|x^{\prime}-x\right|}$. Set $g(t)=$ $f(x+t v), t \in\left[0,\left|x^{\prime}-x\right|\right]$. Note that

$$
g^{\prime}(t)=\partial_{v} f(x+t v)
$$

at any point $t \in\left[0,\left|x^{\prime}-x\right|\right]$ such that $g$ is differentiable at $t$. So we have

$$
c o(g) \subseteq \overline{c o}_{v}(f) .
$$

Recall that in Theorem 3.4 the function $f$ is supposed to be locally Lipschitz. Since any open convex subset of $\mathbf{R}^{n}$ is a union of increasing family of compact convex sets we may assume that $f$ is actually Lipschitz. Hence, by Lemma 4.8 we know that 0 is an extremal point of $\overline{c o}_{v}(f)$. It follows from Lemma 2.1 that 0 is also an extremal point of $c o(g)$. But $c o(g) \neq\{0\}$ since otherwise $f$ is constant on $\left[x, x^{\prime}\right]$. Thus 0 is not in the relative interior of $c o(g)$, so it is enough to apply Lemma 5.4 to obtain Theorem 3.4.

Proof of Theorem 3.9. It goes along the same line as the above proof of Theorem 3.3, however to conclude we use Lemma 4.9.

\section{Proof of Theorems 3.11, 3.13.}

Throughout this section, $f:\left(\mathbf{R}^{n}, 0\right) \rightarrow\left(\mathbf{R}^{n}, 0\right)$ denotes a continuous tame mapgerm. Let $y_{1}, \ldots, y_{n}$ denote a coordinate system of the target. Set $f_{j}=y_{j} \circ$. 
We shall not distinguish between a mapping and its germ. Consider the mapping $\phi_{k}:\left(\mathbf{R}^{n}, 0\right) \rightarrow\left(\mathbf{R}^{n}, 0\right)$, defined by

$$
\left(x_{1}, \ldots, x_{n}\right) \mapsto\left(f_{1}(x), \ldots, f_{k}(x), x_{k+1}, \ldots, x_{n}\right) .
$$

We define $x^{(k)}=\left(x_{1}^{(k)}, \ldots, x_{n}^{(k)}\right), k=1, \ldots, n, n+1$ by the following relations:

$$
x_{j}=x_{j}^{(1)}=\cdots=x_{j}^{(j)}, \quad x_{j}^{(j+1)}=\cdots=x_{j}^{(n+1)}=f_{j}(x), \quad j=1, \ldots, n .
$$

Then the mapping $\phi_{k}$ is expressed by $x \mapsto x^{(k+1)}$. If $\phi_{k-1}$ is a homeomorphism, $x^{(k+1)}$ is considered as a mapping of $x^{(k)}$. In this case, we have the following

Lemma 6.1. $\frac{\partial x_{k}^{(k+1)}}{\partial x_{k}^{(k)}}=\operatorname{det} \frac{\partial\left(f_{1}, \ldots, f_{k}\right)}{\partial\left(x_{1}, \ldots, x_{k}\right)} / \operatorname{det} \frac{\partial\left(f_{1}, \ldots, f_{k-1}\right)}{\partial\left(x_{1}, \ldots, x_{k-1}\right)}$

Proof. Chain rule.

Lemma 6.2. . Consider a continuous tame mapping

$$
f:\left(\mathbf{R}^{n}, 0\right) \rightarrow\left(\mathbf{R}^{n}, 0\right) \quad \text { defined by } \quad\left(x_{1}, \ldots, x_{n}\right) \mapsto\left(f_{1}(x), x_{2}, \ldots, x_{n}\right) .
$$

If there is a positive constant $K$ such that $K \leq \frac{\partial f_{1}}{\partial x_{1}}$, then $f$ is a homeomorphism.

Proof. It is enough to show that $f$ is injective. Let $x \neq x^{\prime}$ be two points in $U$. We set $v=\frac{x^{\prime}-x}{\left|x^{\prime}-x\right|}$. If $v \neq(1,0, \ldots, 0)$, then it is clear that $f(x) \neq f\left(x^{\prime}\right)$. Assume that $v=(1,0, \ldots, 0)$. By Lemma 4.3), we conclude that $\frac{\partial f_{1}}{\partial x_{1}}$ is positive, and $f_{1}(x) \neq$ $f_{1}\left(x^{\prime}\right)$. This completes the proof.

Proof of Theorem 3.11. By the induction on $k$ we show that each $\phi_{k}, k=1, \ldots, n$ is a homeomorphism. By Lemma 6.2, we obtain $\phi_{1}$ is a homeomorphism. Assume that $\phi_{k}$ is a homeomorphism. Then, by Lemma 6.1, we have that

$$
\frac{\partial x_{k+1}^{(k+2)}}{\partial x_{k+1}^{(k+1)}}=\operatorname{det} \frac{\partial\left(f_{1}, \ldots, f_{k+1}\right)}{\partial\left(x_{1}, \ldots, x_{k+1}\right)} / \operatorname{det} \frac{\partial\left(f_{1}, \ldots, f_{k}\right)}{\partial\left(x_{1}, \ldots, x_{k}\right)} \geq \frac{K_{k+1}}{L_{k}} .
$$

Applying Lemma 6.2, we obtain that $\phi_{k+1} \circ \phi_{k}^{-1}$ is a homeomorphism. Thus the mapping $\phi_{k+1}=\left(\phi_{k+1} \circ \phi_{k}^{-1}\right) \circ \phi_{k}$ is a homeomorphism.

For the proof of Theorem 3.11 we shall need a following variant of the previous lemma.

Lemma 6.3. Consider a continuous finite tame mapping

$$
f:\left(\mathbf{R}^{n}, 0\right) \rightarrow\left(\mathbf{R}^{n}, 0\right) \quad \text { defined by } \quad\left(x_{1}, \ldots, x_{n}\right) \mapsto\left(f_{1}(x), x_{2}, \ldots, x_{n}\right) .
$$

If $0 \leq \frac{\partial f_{1}}{\partial x_{1}}$, then $f$ is a homeomorphism.

Proof. It is enough to show that $f$ is injective. Let $x \neq x^{\prime}$ be two points in $U$. We set $v=\frac{x^{\prime}-x}{\left|x^{\prime}-x\right|}$. If $v \neq(1,0, \ldots, 0)$, then it is clear that $f(x) \neq f\left(x^{\prime}\right)$. Assume that $v=(1,0, \ldots, 0)$. By Lemma 4.3 , we conclude that $\frac{\partial f_{1}}{\partial x_{1}}$ is non-negative. Since $f$ is finite, $t \mapsto f_{1}(x+t v)$ is not constant. This completes the proof. 
Proof of Theorem 3.13. Again, by induction on $k$ we show that each $\phi_{k}, k=1, \ldots, n$ is a homeomorphism. From Lemma 6.3, we obtain that $\phi_{1}$ is a homeomorphism. Assume that $\phi_{k}$ is a homeomorphism. Then, by Lemma 6.1, we have that

$$
\frac{\partial x_{k+1}^{(k+2)}}{\partial x_{k+1}^{(k+1)}}=\operatorname{det} \frac{\partial\left(f_{1}, \ldots, f_{k+1}\right)}{\partial\left(x_{1}, \ldots, x_{k+1}\right)} / \operatorname{det} \frac{\partial\left(f_{1}, \ldots, f_{k}\right)}{\partial\left(x_{1}, \ldots, x_{k}\right)} \geq 0 .
$$

Applying Lemma 6.3, we obtain that $\phi_{k+1} \circ \phi_{k}^{-1}$ is a homeomorphism. Thus the mapping $\phi_{k+1}=\left(\phi_{k+1} \circ \phi_{k}^{-1}\right) \circ \phi_{k}$ is a homeomorphism.

\section{Remark on homotopy type of $d f$.}

It looks interesting to investigate the homotopy type of the mapping

$$
d f:\left(\mathbf{R}^{n}-\widehat{B}(f), 0\right) \rightarrow \mathrm{GL}(n, \mathbf{R}),
$$

where $\widehat{B}(f)$ stands for the set of points at which $f$ is not differentiable or the differential exist but is singular. By Cartan-Iwasawa decomposition (see e.g. [8]) we have a homeomorphism

$$
\mathrm{GL}(n, \mathbf{R}) \simeq \mathrm{O}(n) \times \mathbf{R}^{n(n+1) / 2} .
$$

Let $\mathrm{GL}_{+}(n, \mathbf{R})$ denote the set of invertible matrices with positive determinants. Then the above mapping induces a homeomorphism:

$$
\mathrm{GL}_{+}(n, \mathbf{R}) \simeq \mathrm{SO}(n) \times \mathbf{R}^{n(n+1) / 2} .
$$

Let $p: \mathrm{GL}_{+}(n, \mathbf{R}) \rightarrow \mathrm{SO}(n)$ denote the projection to the first component.

Proposition 7.1. Let $f:\left(\mathbf{R}^{2}, 0\right) \rightarrow\left(\mathbf{R}^{2}, 0\right)$ be a continuous tame mapping with $\widehat{B}(f)=\{0\}$ and $f^{-1}(0)=\{0\}$. If the mapping

$$
d f:\left(\mathbf{R}^{2}-0,0\right) \rightarrow \mathrm{GL}(2, \mathbf{R})
$$

is null homotopic, then $f$ is a homeomorphism.

Proof. We may assume that $d f$ is a mapping into $\mathrm{GL}_{+}(2, \mathbf{R})$. Then, by the assumption, the mapping $p \circ d f$ is null homotopic. We remark that $\pi_{1}\left(\mathrm{GL}_{+}(2, \mathbf{R})\right)=$ $\pi_{1}(\mathrm{SO}(2))=\mathbf{Z}$. Since the homotopy type of the mapping

$$
\phi_{f}:\left(\mathbf{R}^{2}-0,0\right) \rightarrow S^{1}, \quad(x, y) \mapsto \frac{f(x, y)}{|f(x, y)|}
$$

determines the topological type of $f$, we can construct a regular homotopy $H_{t}(t \in$ $[0,1])$ between $H_{1}=f$ and $H_{0}$ where $H_{0}$ is the complex function $z^{k}$ for some integer $k$. This homotopy induces a homotopy $p_{\circ} d H_{t}$. Since it is null homotopic, we conclude $k=1$.

The analogous statement is not true for $n \geq 3$, since $\pi_{1}(\mathrm{SO}(n))=\mathbf{Z} / 2 \mathbf{Z}(n \geq 3)$.

Example 7.2. Consider the mapping

$$
f: \mathbf{R}^{3} \rightarrow \mathbf{R}^{3} \quad \text { defined by } \quad(x, y, z) \mapsto \begin{cases}\left(\frac{x\left(x^{2}-3 y^{2}\right)}{x^{2}+y^{2}}, \frac{y\left(3 x^{2}-y^{2}\right)}{x^{2}+y^{2}}, z\right) & (x, y) \neq(0,0) \\ (0,0, z) & (x, y)=(0,0)\end{cases}
$$


This mapping is 3-sheeted covering branching along $z$-axis but $d f: \mathbf{R}^{3}-\widehat{B}(f) \rightarrow$ $\mathrm{GL}(3, \mathbf{R})$ is null homotopic, because $\pi_{1}(\mathrm{SO}(3))=\mathbf{Z} / 2 \mathbf{Z}$.

\section{REFERENCES}

[1] M. Coste, An Introduction to o-minimal Geometry, RAAG Notes, 81 pages, Institut de Recherche Mathématiques de Rennes, November 1999.

[2] F. H. Clarke, On the inverse function theorem, Pacific J. Math. 64 (1976), no. 1, 97-102.

[3] A. Dold, Lectures on algebraic topology, Springer-Verlag, 1972

[4] D. Gale, H. Nikaido, The Jacobian matrix and global univalence of mappings, Math. Annal. 159 (1965), 81-93.

[5] M. S. Gowda, G. Ravindran, Algebraic univalence theorems for nonsmooth functions, J. Math. Anal. Appl. 252 (2000), no. 2, 917-935.

[6] O. Gutú, J. A.. Jaramillo, Global homeomorphisms and covering projections on metric spaces, Math.Ann. 338 (2007), 75-95.

[7] J. Hadamard, Sur les transformations punctuelles, Bull. Soc. Math. France 34 (1906), 71-84.

[8] S. Helgason, Differential Geometry, Lie Groups, and Symmetric Spaces, American Mathematical Society, Providence, R.I., 2001.

[9] J-B. Hiriart-Urruty, C. Lemaréchal, Fundamentals of convex analysis, Grundlehren Text Editions, Springer-Verlag, Berlin, 2001.

[10] A. Gabrielov, Projections of semi-analytic sets, Funct. An. \& Appl. 2 (1968), no. 4, 282-291.

[11] H. Hironaka, Subanalytic sets, in : Number Theory, Algebraic Geometry and Commutative Algebra (in honor of Y. Akizuki) Kinokunya, Tokyo (1973), 453-493.

[12] K. Kurdyka, P. Orro, and S. Simon, Semialgebraic Sard theorem for generalized critical values, J. Diff. Geom. 56, 2000, 67-92.

[13] L. van den Dries, Tame topology and o-minimal structures. London Mathematical Society Lecture Note Series, 248, Cambridge University Press, Cambridge, 1998.

Department of Mathematics, Faculty of Science, Saitama University, 255 ShimoOKubo, URAWA 338-8570, JAPAN

E-mail address: tfukui@rimath.saitama-u.ac.jp

Universite de Savoie et CNRS, UMR 5127 Laboratoire de Mathematiques (LAMA) 73-376 Le BourgeT-DU-LAC CEDEX FRANCE

E-mail address: Krzysztof.Kurdyka@univ-savoie.fr

School of Mathematics and Statistics, University of Sydney, NSW 2006, AusTRALIA

E-mail address: laurent@maths.usyd.edu.au 